\title{
Satellite RNAs interfere with the function of viral RNA silencing suppressors
}

OPEN ACCESS

Edited by:

K. Andrew White,

York University, Canada

Reviewed by:

Juan Antonio Garcia,

Consejo Superior de Investigaciones

Cientificas, Spain

Ricardo Flores,

Instituto de Biología Molecular y Celular de Plantas (UPV-CSIC), Spain

*Correspondence:

Chang-Yong Zhou,

National Citrus Engineering Research

Center, Citrus Research Institute,

Chinese Academy of Agricultural

Sciences, 15 Citrus Village, Xiema,

Beibei, Chongqing 400712, China

zhoucy@swu.edu.cn;

Ming-Bo Wang,

Commonwealth Scientific and

Industrial Research Organisation Plant

Industry, Clunies Ross Street,

Canberra, ACT 2601, Australia

ming-bo.wang@csiro.au

${ }^{\dagger}$ Present Address:

Phil Chi Khang Au,

University of Western Australia,

Faculty of Medicine, Dentistry and Health Sciences, Perth, WA, Australia

Specialty section:

This article was submitted to Virology,

a section of the journal

Frontiers in Plant Science

Received: 20 January 2015 Accepted: 08 April 2015 Published: 24 April 2015

Citation:

Shen W-X, Au PCK, Shi B-J, Smith NA, Dennis ES, Guo H-S, Zhou C-Y and Wang M-B (2015) Satellite RNAs interfere with the function of viral RNA

silencing suppressors.

Front. Plant Sci. 6:281.

doi: $10.3389 /$ fpls.2015.00281

\author{
Wan-Xia Shen ${ }^{1,2}$, Phil Chi Khang Au ${ }^{2 \dagger}$, Bu-Jun Shi ${ }^{3}$, Neil A. Smith $^{2}$, \\ Elizabeth S. Dennis ${ }^{2}$, Hui-Shan Guo ${ }^{4}$, Chang-Yong Zhou ${ }^{1 *}$ and Ming-Bo Wang ${ }^{2 *}$ \\ ${ }^{1}$ National Citrus Engineering Research Center, Citrus Research Institute, Southwest University, Chongqing, China, \\ ${ }^{2}$ Commonwealth Scientific and Industrial Research Organisation Plant Industry, Canberra, ACT, Australia, ${ }^{3}$ Department of \\ Plant Science, Waite Institute, Adelaide University, Glen Osmond, SA, Australia, ${ }^{4}$ State Key Laboratory of Plant Genomics, \\ Institute of Microbiology, Chinese Academy of Sciences, Beijing, China
}

Viral satellite RNAs (satRNAs) are small subviral RNAs and depend on the helper virus for replication and spread. satRNAs can attenuate helper virus-induced symptoms, the mechanism of which remains unclear. Here, we show that two virus-encoded suppressors of RNA silencing (VSRs), Cucumber mosaic virus (CMV) 2b and Tombusvirus P19, suppress hairpin RNA (hpRNA)-induced silencing of a $\beta$-glucuronidase (GUS) gene in Nicotiana benthamiana. This suppression can be overcome by CMV Y-satellite RNA (Y-Sat) via the Y-Sat-derived small interfering RNAs (siRNAs), which bind to the VSRs and displace the bound hpGUS-derived siRNAs. We also show that microRNA target gene expression in $N$. tabacum was elevated by CMV infection, presumably due to function of the $2 \mathrm{~b}$ VSR, but this upregulation of microRNA target genes was reversed in the presence of Y-Sat. These results suggest that satRNA infection minimizes the effect of VSRs on host siRNA and microRNA-directed silencing. Our results suggest that the high abundance of satRNA-derived siRNAs contributes to symptom attenuation by binding helper virus-encoded VSRs, minimizing the capacity of the VSRs to bind host siRNA and miRNA and interfere with their function.

Keywords: virus-encoded suppressor, satellite RNA, symptom attenuation, RNA silencing, microRNA expression

\section{Introduction}

SatRNAs (satRNAs) depend on their associated viruses (helper viruses) for replication, encapsidation, systemic movement and transmission, and are regarded as parasites of their helper viruses. They have small RNA genomes sized from 220 to 1400 nucleotides (nt), which share little or no sequence homology with the genome of helper viruses (Roossinck et al., 1992; Simon et al., 2004). The relatively large (800-1400 nt) satRNAs can encode one or a few proteins, but the majority of satRNAs, with $<700 \mathrm{nt}$ genome, possess no mRNA activity and do not encode any protein (Roossinck et al., 1992; Simon et al., 2004). Some satRNAs can induce specific symptoms in virus-infected plants (Garcia-Arenal and Palukaitis, 1999; Hu et al., 2009), for example, yellowing symptoms in Nicotiana species induced by Y satRNA (Y-Sat) of Cucumber mosaic virus (CMV) (Masuta and Takanami, 1989). Recent studies showed that the yellowing symptoms are the result of the silencing of the chlorophyll biosynthetic gene CHLI induced by Y-Sat-derived small interfering RNAs (siRNAs), which have a 22-nt sequence homologous to CHLI (Shimura et al., 2011; Smith et al., 2011). In contrast to Y-Sat, most satRNAs do not induce their own 
symptoms. Another feature of satRNAs is the attenuation of helper virus-induced symptoms (Roossinck et al., 1992; Simon et al., 2004). This property has been used in transgenic plants for viral disease prevention (Baulcombe et al., 1986; Gerlach et al., 1987; Harrison et al., 1987). However, how satRNAs attenuate the symptoms remains a matter for debate. It was proposed that satRNAs might compete with the helper viruses for RNA replicase or increase antiviral silencing against the accumulation of the helper viruses (Piazzolla et al., 1982; Wu and Kaper, 1995; Hou et al., 2011). However, in some cases, satRNAs attenuate the symptoms but do not reduce the accumulation of helper viruses (Harrison et al., 1987; Moriones et al., 1992). Therefore, other unknown factors may also be involved in the regulation of helper virus-induced symptoms.

RNA silencing is a sequence-specific RNA degradation process induced by 21-24-nt small RNAs (sRNAs) processed from double-stranded RNA (dsRNA) or self-complementary hairpin RNA (hpRNA) by Dicer or Dicer-like protein, an RNase III-like endoribonuclease. One strand of each sRNA is loaded onto the RNA-induced silencing complex (RISC) and guides Argonaute (AGO) proteins in the RISC to cleave targeted RNA (Zamore, 2001). In plants RNA silencing has been recognized as an antiviral defense mechanism by which siRNAs derived from the virus guide the degradation of the viral RNA in the infected plant (Baulcombe and Molnar, 2004; Ruiz-Ferrer and Voinnet, 2009). To overcome this host defense mechanism, viruses have evolved a counter-defense strategy by encoding suppressors of RNA silencing (VSRs) (Zhang et al., 2006; Shimura and Pantaleo, 2011; Pumplin and Voinnet, 2013). A predominant action mode of VSRs is to bind the siRNAs preventing their entry into the RISC and degradation of the viral RNA (Lakatos et al., 2006). Beside viral siRNAs, VSRs also interfere with the function of host small RNAs including microRNAs (miRNAs) (Kasschau et al., 2003; Chapman et al., 2004; Zhang et al., 2006; Lewsey et al., 2007). miRNAs play a critical role in processes such as cell division, leaf formation, and flower development in plants (Voinnet, 2009). Therefore, while VSRs help the viruses to evade antiviral RNA silencing by the host, they can also induce symptoms by disrupting functions of specific miRNA species, which regulate host plant development (Zhang et al., 2006; Lewsey et al., 2007; Jay et al., 2011; Du et al., 2014).

In this study, we used an Agrobacterium infiltration (agroinfiltration)-based transient expression system to investigate how the satRNA of CMV Y (Y-Sat) affected the ability of CMVencoded VSR $2 \mathrm{~b}$ and Tombusvirus-encoded VSR P19 to suppress hpRNA-induced gene silencing in Nicotiana benthamiana. Both VSRs suppressed hpRNA-induced gene silencing, but in the presence of Y-Sat, this VSR effect was reversed. Using RNA immunoprecipitation, we showed that this reversion is due to the bound hpRNA-derived siRNAs being displaced from the VSRs by YSat-derived siRNAs. We also found that Y-Sat infection minimizes the over-expression of target genes of endogenous miRNAs caused by CMV infection, indicating that Y-Sat inhibited the effect of the $2 b$ VSR on the function of host miRNAs.

\section{Materials and Methods}

\section{Plant Growth, Virus Inoculation, Plasmid Constructs, and Plant Transformation}

$N$. benthamiana and $N$. tabacum (HcPro transgenic; Wang et al., 2004) plants were grown at $25^{\circ} \mathrm{C}$ under a 16-h light/8-h dark cycle. For virus infection, leaves of 3-5 week old plants were dusted with carborundum and rub-inoculated with leaf extracts of previously infected plants in $0.1 \mathrm{M}$ phosphate buffer ( $\mathrm{pH}$ 7.2).

The constructs of GFP and P19 driven by the CaMV 35S promoter were previously described (Wood et al., 2009). The $2 b$ (wild-type) and $2 b m$ (mutant; created by substituting "C" for " $U$ " in the start codon AUG, as well as three other AUG codons present in the $2 \mathrm{~b}$ coding sequence) constructs were also previously described (Hou et al., 2011). The GUS construct (pIG121Hm) contains an intron in the coding sequence, which was designed to prevent GUS expression in bacterial cells (Ohta et al., 1990; Ishikawa, 2009). The $h p G U S$ construct was previously reported, designed to express a long GUS hairpin RNA with a 563 bp dsRNA arm and an 1113 nt loop (Wang and Waterhouse, 2000). All of the above constructs were introduced into A. tumefaciens strains AGL1 (GFP and $h p G U S$ ) or GV3101 (pIG121Hm, $2 b, 2 b m$, and P19).

\section{Agrobacterium Infiltration Assay}

A. tumefaciens strains containing plant expression constructs were grown overnight at $28^{\circ} \mathrm{C}$ in Luria-Bertani medium (LB) containing appropriate antibiotics. After centrifugation, Agrobacterium cells were re-suspended in buffer containing $10 \mathrm{mM} \mathrm{MgCl} 2$ and $150 \mu \mathrm{g} / \mathrm{mL}$ acetosyringone, with a final optical density at $600 \mathrm{~nm}\left(\mathrm{OD}_{600}\right)$ of 0.7 . The bacterial suspensions were incubated at room temperature for $\sim 3 \mathrm{~h}$ and then infiltrated into expanded $N$. benthamiana leaves using flat-pointed syringe (Liu and Lomonossoff, 2002). For protein and RNA isolation, agro-infiltrated leaf sections were visualized under a blue light torch (NightSea ${ }^{\mathrm{TM}}, \mathrm{DFP}-1^{\mathrm{TM}}$, for exciting green light emission), excised with a pair of scissors and immediately frozen in liquid nitrogen at 4 or 5 days post agro-infiltration (dpa).

\section{MUG Assay of GUS Expression}

For fluorometric MUG (4-methyl-umbelliferyl- $\beta$-Dgulcuronide) assay, agro-infiltrated leaf sections were homogenized in extraction buffer $(50 \mathrm{mM}$ sodium phosphate, $10 \mathrm{mM} \mathrm{Na}{ }_{2}$ EDTA, $0.1 \%$ sarcosyl, $10 \mathrm{mM} \beta$-mercaptoehtanol, $\mathrm{pH}$ 7.0). The homogenates were subjected to centrifugation for $5 \mathrm{~min}$ at $4^{\circ} \mathrm{C}$ at $16,000 \times \mathrm{g}$, and the supernatants were collected and kept on ice. Concentration of total protein was determined using the Bradford reagent. MUG reactions were carried out at $37^{\circ} \mathrm{C}$ and GUS activities were calculated essentially as previously described (Chen et al., 2005) using a fluorescence plate reader (Wallac 1420 VICTOR, PerkinElmer, Turku, Finland). 


\section{RNA Isolation, Northern Blot Analysis, and RT-PCR}

Total RNA was isolated from N. benthamiana and N. tabacum leaves or leaf sections using Trizol reagent (Invitrogen) following the manufacturer's instructions. Isopropanol precipitation of the total RNA was carried out overnight at $-20^{\circ} \mathrm{C}$ to maximize recovery of the small RNA fraction.

For detection of large RNAs in northern blot hybridization, total RNA $(\sim 5-10 \mu \mathrm{g})$ was separated in formaldehyde-agarose gel (Sambrook et al., 1989) and blotted onto Hybond-N membrane (Amersham Life Science, NSW, Australia) in $10 \times$ SSC. The membrane was hybridized with $\left[\alpha-{ }^{32} \mathrm{P}\right]$ UTP-labeled RNA probes obtained by in vitro transcription using radioactive UTP from the respective sequences (Supplemental Text 1) cloned into pGEM plasmids (Promega, NSW, Australia). Hybridization was performed as previously described (Wang et al., 2001).

For detection of small RNAs in northern blot hybridization, approximately $10-20 \mu \mathrm{g}$ of total RNA were separated in a denaturing (urea) $17 \%$ polyacrylamide gel. RNA was electroblotted onto a Hybond- $\mathrm{N}^{+}$membrane (Amersham Life Science) in $0.5 \times$ TBE buffer, UV cross-linked, and hybridized overnight at $42^{\circ} \mathrm{C}$ with $\left[\alpha-{ }^{32} \mathrm{P}\right]$ UTP labeled, carbonate buffer-fragmented RNA probe (Wang et al., 2001). The hybridized membrane was washed three times $\left(20 \mathrm{~min}\right.$ per wash) at $40^{\circ} \mathrm{C}$ with $2 \times$ SSC containing $0.2 \%$ SDS. Both large and small RNA northern blots were visualized on a phosphoimager (Fujifilm FLA 5000, Tokyo, Japan).

For RT-PCR or qRT-PCR analysis of $2 \mathrm{~b}$, HcPro mRNA, or miRNA target genes, total RNA $(\sim 1 \mu \mathrm{g})$ isolated from infected and uninfected leaves was reverse-transcribed with Superscript III reverse transcriptase (Invitrogen) following the manufacturer's instruction using oligo- $\mathrm{dT}_{23}$ as primer. The synthesized cDNA was amplified using 2b, HcPro or miRNA target genespecific primers (see Supplemental Text 2 for primer sequences) with $56^{\circ} \mathrm{C}(2 \mathrm{~b})$ or $60^{\circ} \mathrm{C}$ (HcPro and miRNA target genes; qRTPCR) as the annealing temperature. PCR cycle numbers (2b) are given on the gel pictures. Real-time PCR was performed in the Rotor-Gene 6000 (Corbett Life Science, San Francisco, USA) real-time rotary analyser using SYBR Green reagent and Platinum Taq polymerase (Invitrogen) in three technical replicates for each sample. mRNA encoding the $N$. tabacum elongation factor $1 \alpha($ EF1 $\alpha)$ was amplified for use as loading control.

\section{RNA Immunoprecipitation}

RNA immunoprecipitation was performed as described by Omarov et al. (2006) with minor changes. Briefly, tissues $(2 \mathrm{~g})$ were ground into fine powder in liquid nitrogen and re-suspended in $2 \mathrm{~mL}$ ice-cold extraction buffer with $150 \mathrm{mM}$ HEPES (pH 7.5), $200 \mathrm{mM} \mathrm{NaCl}, 1 \mathrm{mM}$ EDTA, $2 \mathrm{mM}$ DTT, 16 unit RNaseOUT (Invitrogen, Victoria, Australia) and $20 \mu \mathrm{L}$ Plant protease inhibitors (Sigma-Aldrich, NSW, Australia). The homogenized plant material was filtered through two layers of miracloth (Calbiochem, Victoria, Australia) and centrifuged twice at $10,000 \times \mathrm{g}$ at $4^{\circ} \mathrm{C}$ for $15 \mathrm{~min}$. The supernatant was precleared in $10 \mu \mathrm{L}$ Salmon Sperm DNA/Protein G agarose beads (Millipore, Victoria, Australia) for $30 \mathrm{~min}$ at $4{ }^{\circ} \mathrm{C}$ under constant rotation. An aliquot $(800 \mu \mathrm{L})$ of pre-cleared lysate was then immunoprecipitated with $2 \mu \mathrm{L}$ of P19 rabbit antibodies (A gift from Prof. Herman Bertus Scholthof, Texas A\&M University) and $30 \mu \mathrm{L}$ of Salmon Sperm DNA/Protein G agarose beads (Millipore) for $3 \mathrm{~h}$ at $4^{\circ} \mathrm{C}$ under constant rotation. Following immunoprecipitation, the agarose-antibody-P19/RNA complexes were washed six times in extraction buffer. P19/RNA complexes were eluted with $100 \mu \mathrm{L}$ of RNA-IP elution buffer [100 mMTris- $\mathrm{HCl} \mathrm{pH}$ 8.0, 10 mM EDTA, 1\% SDS, 40 units of RNaseOUT (Invitrogen), $20 \mu \mathrm{g}$ of Proteinase K (Sigma-Aldrich)] at $65^{\circ} \mathrm{C}$ for $1 \mathrm{~h}$. RNA was purified by extraction with an equal volume of acidic phenol/chloroform (Ambion, Applied Biosystems, Victoria, Australia), followed by overnight ethanol precipitation in the presence of acidic sodium acetate and $20 \mu \mathrm{g}$ glycogen (Fermentas, Thermo fisher scientific, Victoria, Australia) at $-80^{\circ} \mathrm{C}$, and resuspended in DEPC-treated $\mathrm{H}_{2} \mathrm{O}$ for small RNA northern blot hybridization. For Western blot analysis of the pull-down material, elution step was omitted and washed beads were stored at $-20^{\circ} \mathrm{C}$ for Western blotting.

\section{Western Blot Analysis}

Beads containing the immunoprecipitated P19/RNA complexes were denatured at $95^{\circ} \mathrm{C}$ for $5 \mathrm{~min}$ with addition of Western blot loading buffer (Bromophenol blue, $0.125 \mathrm{M}$ Tris- $\mathrm{HCl}, 4 \%$ SDS, $20 \%$ glycerol, $2 \% \beta$-mercaptoethanol, $\mathrm{pH} 6.8$ ). The proteins were separated on a $15 \%$ SDS polyacrylamide gel, transferred to Immobilon P membrane (Millipore) and detected by chemiluminescence using 1/2500 anti-P19 antibody (Omarov et al., 2006) and 1/5000 anti-rabbit Ig HRP conjugate (Chemicon, Victoria, Australia).

\section{Results}

\section{Y-Sat Reduces the Symptoms Caused by the Helper Virus Fny-CMV}

The strains of Cucumber mosaic virus (CMV) are classified into two subgroups, I and II, based on their serology, symptomatology and sequence homology (Palukaitis et al., 1992). Fny-CMV is a subgroup I strain and induces severe symptoms in many host plant species (Zhang et al., 1994). Its encoded VSR 2b has been shown to be a strong virulence contributor (Shi et al., 2002). In $N$. benthamiana, Fny-CMV not only induced severe symptoms, but also constrained plant growth (Figure 1A). However, in the presence of Y-Sat, the severity of the Fny-CMV-caused symptoms was reduced and plant growth improved, despite the bright yellow symptoms typical of Y-Sat infection (Figure 1A). This demonstrates that Y-Sat attenuates the symptoms induced by Fny-CMV, which is consistent with previous reports (Hou et al., 2011). Northern blot hybridization showed that Y-Sat coinfection reduced the level of CMV RNA, but not dramatically (Figure 1B).

\section{Y-Sat is the Dominant Source of Viral siRNAs in Infected Plants}

Previous studies by northern blotting and small-scale sequencing showed that the replication of Y-Sat is accompanied by high levels of sRNAs derived from Y-Sat (Wang et al., 2004; Ebhardt and Unrau, 2009). To further demonstrate this, we obtained 

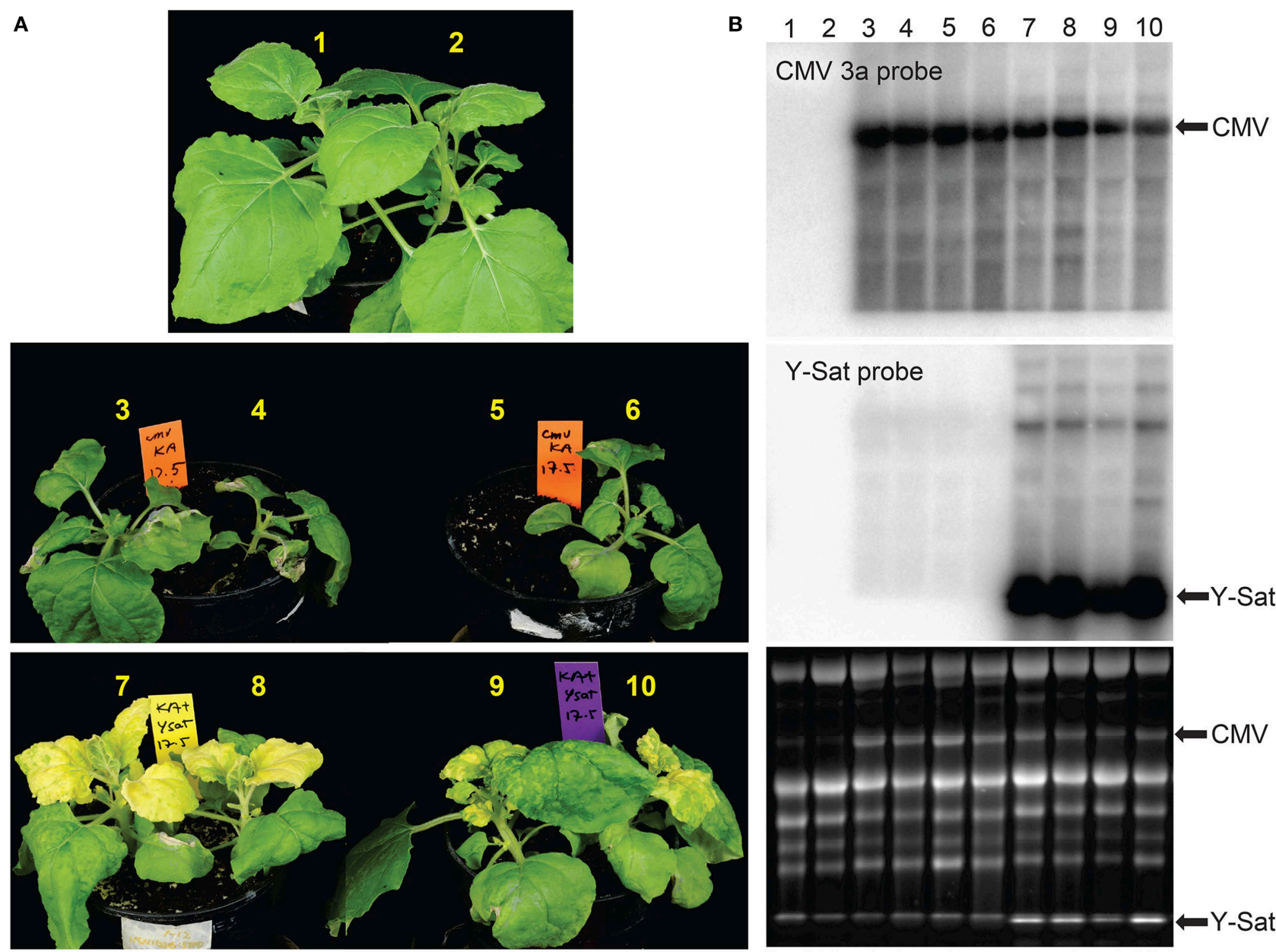

C

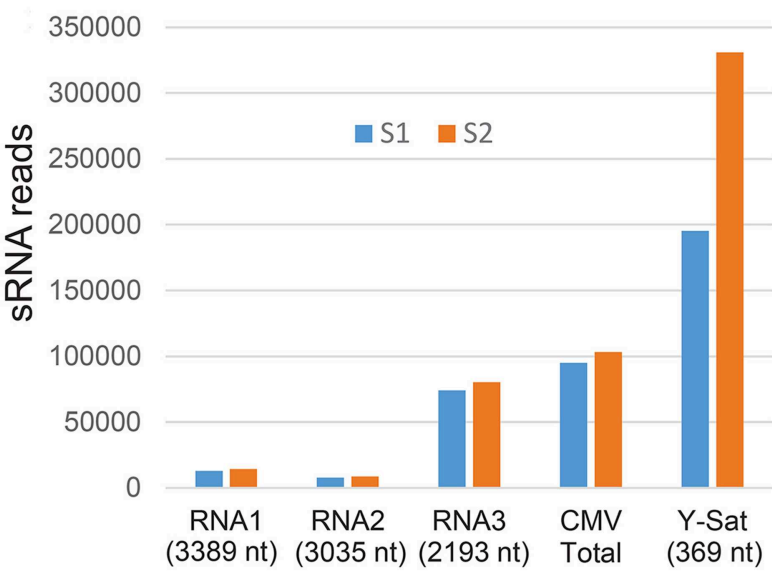

FIGURE 1 | Y-Sat infection reduces the severity of CMV-caused symptoms and is associated with high abundance of Y-Sat siRNAs. (A) $N$. benthamiana plants co-infected with both Fny-CMV and Y-Sat (bottom) (12 days post inoculation) are healthier and bigger than those infected with Fny-CMV alone (middle), despite the Y-Sat-specific bright yellowing symptoms. Note that plant \#5 was very weak and accidentally uprooted prior to photographing. (B) Northern blot analysis of plants in (A), showing high amounts of Fny-CMV RNA in both CMV
D 300000

$=20 \mathrm{nt} \quad-21 \mathrm{nt} \quad-22 \mathrm{nt} \quad-23 \mathrm{nt} \quad-24 \mathrm{nt}$

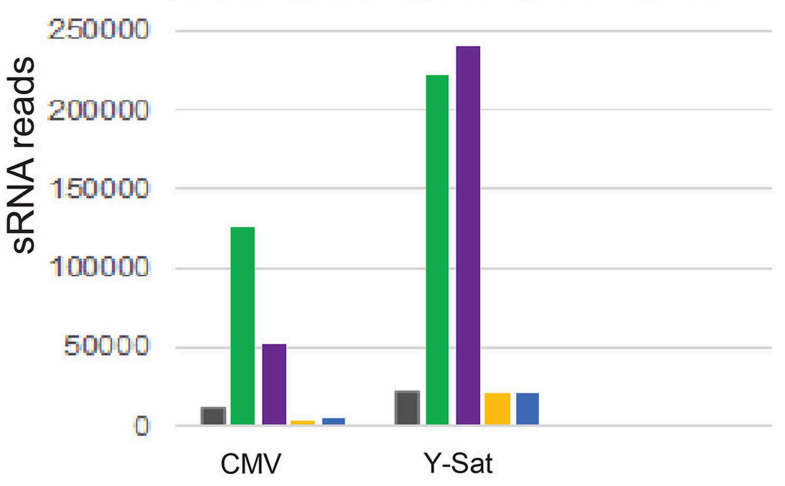

and $\mathrm{CMV}+\mathrm{Y}$-Sat-infected plants, although CMV RNA abundance is in general reduced in Y-Sat-infected plants. The CMV RNA3 and Y-Sat genomic RNA bands are indicated in both the hybridized northern blot (top and middle) and the stained RNA gel (bottom). (C) Total number of 20-24 nt sRNA reads perfectly mapped to Q-CMV (RNA1, RNA2, RNA3, and whole genome) and Y-Sat in two Q-CMV+Y-Sat-infected

N. tabacum samples (S1 and S2). (D) Size distribution of Q-CMV and Y-Sat-derived SRNAs. 
two sets of sRNA sequence data from N. tabacum plants coinfected with Q-CMV (a subgroup II CMV) and Y-Sat using Illumina deep sequencing technology, and analyzed the relative abundance of Y-Sat and CMV-derived sRNAs. A total of 195,291 and 330,965 reads of 20-24 nt sRNAs (from a total of approximately 8 million sRNA reads each) were perfectly mapped to the Y-Sat genome in the two samples S1 and S2, respectively (Figure 1C). These numbers are approximately 2 and 3 times as much as the total 20-24 nt sRNA reads perfectly mapped to the Q-CMV genome (95,073 and 103,443 for S1 and S2, respectively) (Figure 1C). This is despite the 23 fold bigger size of the CMV genome (8617 nt) than the Y-Sat genome (369 nt), indicating that the Y-Sat genome has approximately 50 fold more sRNA coverage than the CMV genome, which is consistent with the previously reported small-scale sequencing result (Ebhardt and Unrau, 2009). This relatively high abundance of Y-Sat sRNAs could result from a relatively high proportion of Y-Sat RNA in the total viral RNA population of the Q-CMV+Y-Sat-infected N. tabacum plants, but a previous report showed that Y-Sat RNA represents about $31-38 \%$ of the total viral RNAs (Takanami, 1981), well below the $2 \sim 3$ fold level of Y-Sat sRNAs.

\section{Y-Sat RNA Interferes with the Function of $2 b$ in Nicotiana benthamiana Leaves}

Cucumoviral $2 \mathrm{~b}$ proteins are essential for cucumoviruses to cause viral symptoms (Ding et al., 1996; Shi et al., 2002; Soards et al., 2002; Zhang et al., 2006; Lewsey et al., 2007), while Y-Sat infection reduces the symptoms (Figure 1). This led us to investigate if $\mathrm{Y}$-Sat infection interferes with the $2 \mathrm{~b}$ functions. We developed an Agrobacterium infiltration (agro-infiltration)based transient expression system. In this system equal amounts of VSR-expressing constructs were agro-infiltrated into N. benthamiana leaves with infection of Q-CMV or Q-CMV plus Y-Sat, and mock-treated plant leaves, and the level of hpRNA-induced silencing of the $\beta$-glucuronidase (GUS) reporter gene in the leaves was measured. We used the subgroup II CMV strain, Q-CMV, as the helper virus to infect $N$. benthamiana instead of Fny-CMV. This was because Q-CMV encodes a relatively weak 2b VSR (Chapman et al., 2004; Zhang et al., 2006; Ye et al., 2009) and the level of VSR activity from the infecting Q-CMV is negligible compared to that from the agro-infiltrated VSR constructs expressing a strong VSR (SD-CMV $2 \mathrm{~b}$ and tombusvirus P19) at relatively high levels. This allowed us to examine the effect of Y-Sat specifically on the agro-infiltrated VSR. In addition, Q-CMV only induces mild symptoms in N. benthamiana and allowed the normal leaf expansion required for agro-infiltration. Furthermore, Q-CMV, like Fny-CMV, supports high levels of Y-Sat replication and Y-Sat siRNA accumulation (Figure $1 \mathrm{C}$ and Wang et al., 2004). The following constructs were used in the agro-infiltration assay: GUS reporter gene (GUS), GUS silencinginducing hpRNA ( $h p G U S$ ), strong 2b (2b) VSR encoded by the subgroup I Shan-Dong (SD)-CMV (Hou et al., 2011), and green florescent protein gene $(G F P)$ as a visual marker. A construct expressing a mutant $2 \mathrm{~b}$ gene $(2 \mathrm{bm})$, in which the start codon and three other in-frame AUG codons of the $2 \mathrm{~b}$ coding sequence were mutated (Duan et al., 2012), was used as a negative control. Gene expression in all of these constructs was driven by a constitutive Cauliflower mosaic virus (CaMV) 35S promoter.

As shown by the fluorometric MUG (4-methylumbelliferyl$\beta$-D-glucuronide) assay in Figure $2 \mathrm{~A}$, without $2 b$ co-infiltration, strong GUS silencing was induced by $h p G U S$ in all uninfected (mock), Q-CMV-infected (Q-CMV), and Q-CMV+Y-Sat coinfected plants. Northern blotting confirmed that the GUS gene was silenced at the mRNA level (Figure 2B, top two panels). When GUS and $h p G U S$ were co-infiltrated with $2 \mathrm{bm}$, GUS silencing was not suppressed (Figures 2A,B). However, when GUS and $h p G U S$ were co-infiltrated with $2 b$, the GUS activity was recovered substantially in the mock and Q-CMV-infected plants, but this was not the case for Q-CMV+Y-Sat co-infected plants, which continued to show strong GUS silencing (Figures 2A,B). These results indicate that $2 \mathrm{~b}$ possesses the ability to suppress the hpRNA-induced GUS silencing but this ability is greatly inhibited in the presence of Y-Sat. There were variations in CMV RNA3 levels among the different samples, with a few samples containing particularly high amounts of CMV RNA3, although the four $G U S+h p G U S+2 b$-infiltrated samples showed relatively uniform levels (Figure 2B). The variations could result from different age or infection state of the leaf tissues harvested because they did not seem to be due to the presence or absence of Y-Sat. CMV-infected samples did not show enhanced GUS expression in comparison to mock-treated ones (Figure 2A), and the variations in CMV RNA3 level did not have a clear effect on GUS expression levels (Figure 2B), presumably because of the weak VSR activity of the Q-CMV 2b.

Northern blot analysis of sRNAs corresponding to the 563-bp dsRNA arm region of the $h p G U S$ transcript (Wang and Waterhouse, 2000) showed that Y-Sat did not increase the accumulation of $h p G U S$-derived siRNAs in the absence (Figure $2 \mathrm{C}$, lanes $3-6)$ or presence (lanes 7-10) of $2 b$. This indicates that the enhanced GUS silencing upon Y-Sat infection was not caused by an increase in the amount of $h p G U S$-derived siRNAs. In addition, a semi-quantitative RT-PCR analysis showed that the expression level of the agro-infiltrated $2 b$ gene was not affected by Q-CMV or Q-CMV+Y-Sat infection (Supplemental Figure S1). These results indicate that Y-Sat minimized the suppression of the hpRNA-induced GUS silencing mediated by $2 \mathrm{~b}$.

Surprisingly, when a probe complementary to the loop of the hpGUS RNA was used, an intense band of the predicted loop size $(\sim 1.1 \mathrm{~kb})$ was detected in the $2 b$-infiltrated tissues (Figure 2B, 2nd panel; lanes 13-16), indicating that the $2 \mathrm{~b}$ protein enhanced the accumulation of the hpRNA loop. Loop fragments have been known to accumulate in hpRNA-expressing plants (Wang et al., 2008). However, the intensity of the hybridized loop fragment from the tissues infected with Q-CMV+Y-Sat was much weaker than that from the tissues infected with Q-CMV alone (lanes 15-16 vs. lanes 13-14). This further suggests that Y-Sat interferes with $2 \mathrm{~b}$ function.

\section{Y-Sat RNA Interferes with VSR P19 Function in N. benthamiana Leaves}

The tombusvirus-encoded P19 is a well-characterized VSR that suppresses RNA silencing via binding and sequestering sRNAs (Vargason et al., 2003; Baulcombe and Molnar, 2004; Lakatos 


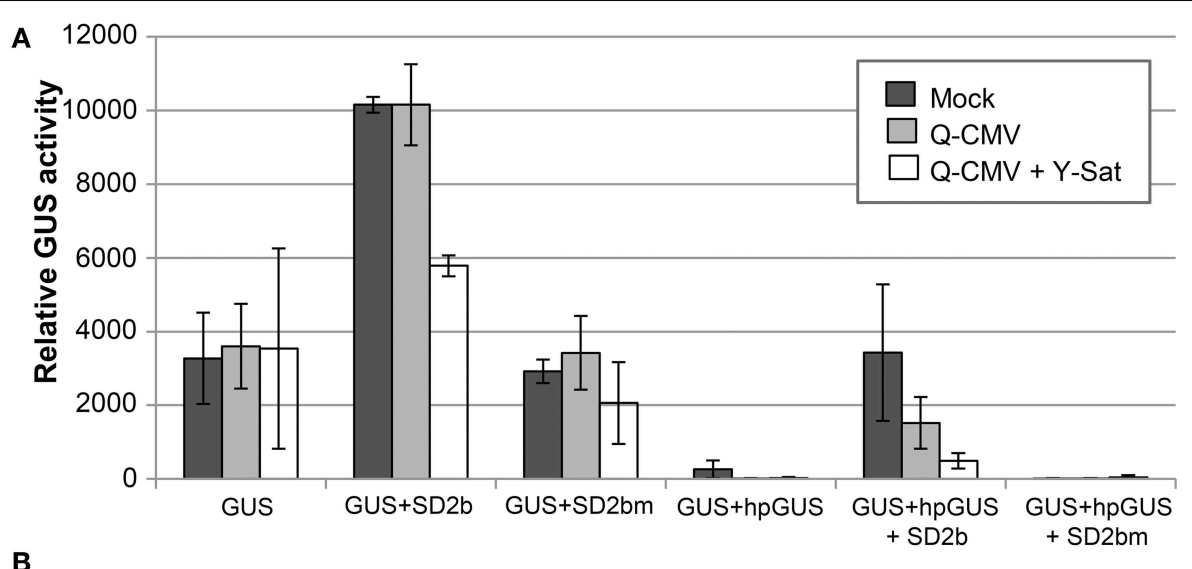

B
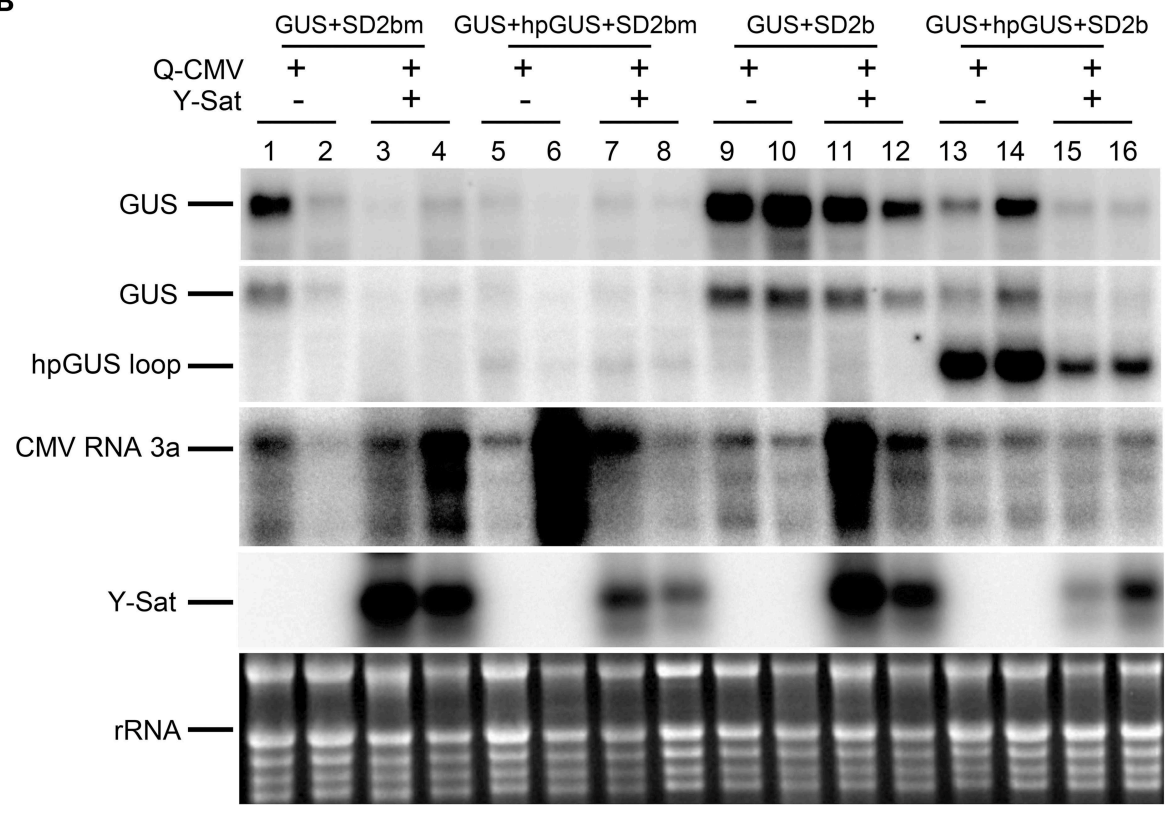

C
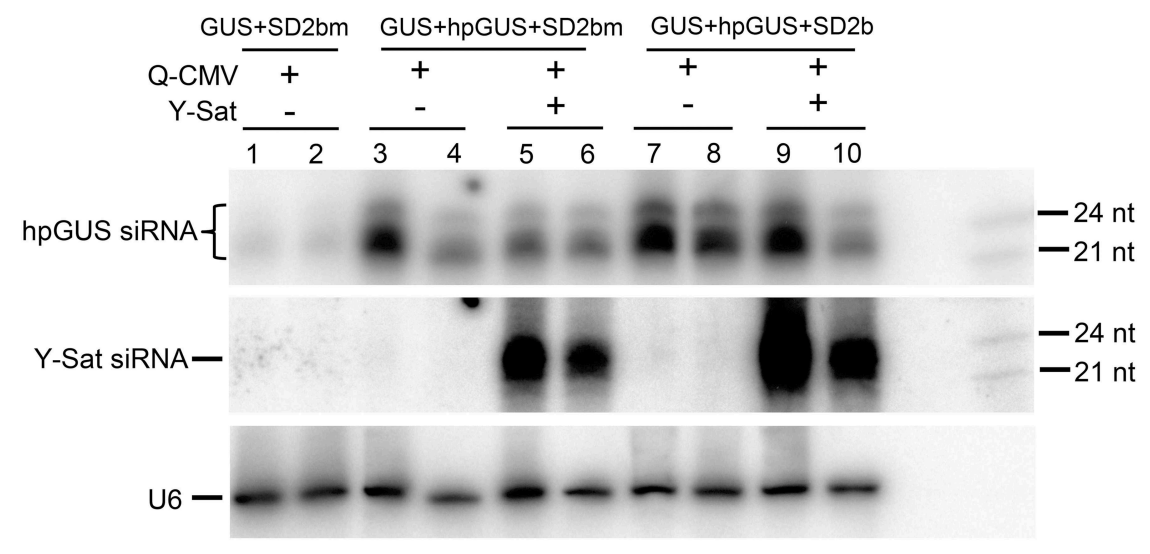

FIGURE 2 | The suppression of hpRNA-induced GUS silencing by agro-infiltrated $2 \mathrm{~b}$ is released by $\mathbf{C M V}+\mathbf{Y}$-Sat infection. (A) MUG assay of GUS expression in Mock (inoculated only with buffer), Q-CMV and $\mathrm{Q}-\mathrm{CMV}+\mathrm{Y}$-Sat-infected $\mathrm{N}$. benthamiana leaves infiltrated with the constructs indicated. The plants were infiltrated at 11 or 13 days after inoculation. The GUS activity was analyzed at 4 days post agroinfiltration (dpa). (B) Northern blot hybridization analysis of GUS expression in a subset of the samples described in (A). Ten microgram of total RNA from each sample was hybridized with an antisense GUS probe to detect GUS mRNA (top panel), a probe complementary to the loop of the hpGUS RNA to detect both GUS mRNA and hpGUS loop fragments (second panel), a CMV RNA 3a probe to detect CMV (third panel), and a Y-Sat probe to detect Y-Sat (forth panel) in the agroinfiltrated tissues. Ethidium bromide-stained rRNA bands are used as a loading control (bottom panel). (C) Northern blot hybridization analysis of small RNAs in a subset of the samples described in (A). Twenty microgram (Continued) 
FIGURE 2 | Continued

of total RNA each was hybridized with a RNA probe corresponding to the dsRNA stem region of hpGUS to detect hpGUS-derived siRNA (top panel), an antisense Y-Sat RNA probe to detect Y-Sat-derived siRNAs (middle panel) and a U6 probe to show loading control (bottom panel).
The 21 and 24-nt sRNA size markers are loaded in the last lane on the right. As can be seen, the level of hpGUS-derived siRNAs is not significantly affected by Q-CMV Y-Sat infection. SD2b or SD2bm, wild-type or mutant $2 \mathrm{~b}$ derived from the group I Shandong (SD) strain of CMV. et al., 2006). We examined if Y-Sat also interferes with P19 functions. The same agro-infiltration system used above for 2b was applied. P19 suppressed $h p G U S$-induced GUS silencing in mock and Q-CMV-infected N. benthaminana tissues, and the suppression was negated in Q-CMV+Y-Sat-infected tissues (Figures 3A,B). In addition, the presence of Y-Sat did not increase the accumulation of $h p G U S$-derived siRNAs (Figure 3C), indicating that the recovery of GUS silencing in $\mathrm{Q}-\mathrm{CMV}+\mathrm{Y}-\mathrm{Sat}$-infected tissues was not due to an increase in the amount of $h p G U S$-derived siRNAs. These results indicate that Y-Sat enhances hpRNA-induced silencing in the agro-infiltrated $N$. benthamiana leaves by interfering with the function of P19 minimizing P19-mediated suppression of silencing. Thus, Y-Sat may use the same mechanism to interfere with the functions of $2 \mathrm{~b}$ and P19.

It is worth mentioning that both $2 \mathrm{~b}$ and P19 enhanced GUS expression in the GUS-infiltrated plants lacking $h p G U S$ (Figures 2A,B and Figures 3A,B; compare "GUS" or "GUS+2bm" with "GUS+2b" or "GUS+P19"). This probably resulted from VSR-mediated suppression of sense co-suppression or posttranscriptional gene silencing that occurs to agro-infiltrated transgenes in N. benthamiana leaves (Voinnet et al., 1999). In the presence of Y-Sat, part of the enhancement of GUS expression by 2b or P19 was lost (Figures 2B, 3B; compare lanes 11-12 with lanes 9-10), further supporting the conclusion that Y-Sat interferes with the function of $2 b$ and P19.

\section{Y-Sat Infection Minimizes the Effect of CMV Infection on Expression of miRNA Target Genes}

VSRs, including CMV 2b, suppress the function of both viral and host siRNAs and miRNAs (Brigneti et al., 1998; Kasschau et al., 2003; Pumplin and Voinnet, 2013). Our agro-infiltration assays indicated that Y-Sat infection interferes with the VSR effect on siRNA-directed gene silencing in the host plant. To determine if Y-Sat infection affected the function of VSRs on host miRNAdirected gene silencing, expression levels of target genes of five miRNAs (miR156, miR160, miR168, miR172, and miR395) in the Q-CMV and Q-CMV+Y-Sat-infected transgenic N. tabacum plants overexpressing potyvirus (Tobacco etch virus)-encoded VSR HcPro were analyzed. The HcPro plants rather than wildtype plants were used for two reasons. First, the plants do not develop yellowing symptoms with Q-CMV+Y-Sat infection, presumably because Y-Sat siRNA-directed silencing of the chlorophyll biosynthetic gene CHLI is repressed by HcPro (Shimura et al., 2011; Smith et al., 2011; Shen et al., 2014). This minimizes the possible effect of the yellowing symptoms on the expression of miRNAs' targets. Second, infection of the HcPro plants, but not wild-type plants, with Q-CMV resulted in consistent upregulation of miRNA target gene expression (Figure 4A and data not shown), possibly because of a synergistic effect between CMV $2 b$ and HcPro or the relatively high level of CMV accumulation in HcPro plants in comparison to wild-type plants. This allowed us to examine the effect of Y-Sat on VSR-mediated mis-regulation of miRNA target genes. It is worth noting that HcPro increases the expression levels of several miRNAs' targets in Arabidopsis (Kasschau et al., 2003), but among the five N. tabacum miRNA target genes examined, only that of miR160 appeared to be upregulated in the HcPro-expressing N. tabacum plants without CMV infection (Figure 4A). The target genes of the remaining four miRNAs were expressed at similar levels between HcPro-expressing (HcPro+) (samples 3-4) and wild-type (HcPro-) (samples 1-2) N. tabacum plants (Figure 4A).

All five miRNA target genes showed increased expression in the CMV-infected HcPro+ plants compared to the uninfected HcPro+ plants (Figure 4A, samples 5-6 vs. 3-4). This suggests that the $2 \mathrm{~b}$ protein from the infecting $\mathrm{CMV}$, probably in conjunction with the transgene-expressed HcPro, suppressed miRNAdirected silencing resulting in upregulation of the miRNA target genes in the CMV-infected plants. However, in the plants coinfected with CMV and Y-Sat, the expression levels of these target genes were reduced and became similar to those in the uninfected HcPro+ plants (compare samples 7-8 with samples 5-6 or 3-4). This change was not due to a change in HcPro expression because its expression level was similar between the CMV and $\mathrm{CMV}+\mathrm{Y}$-Sat-infected plants and showed no correlation with the expression of miRNA target genes (Figure 4B). Furthermore, this change was not caused by a change in CMV RNA level because there was in general no correlation between the expression level of miRNA target genes and the amount of CMV RNA in the CMV and CMV+Y-Sat-infected plants (Figures 4A,C). The expression of the miR156 target gene in the two CMV-infected plants appeared to be an exception, which showed a positive correlation with the amount of CMV RNA (Figures 4A,C). Taken together, the decrease in miRNA target gene expression upon Y-Sat infection suggests that the suppression of miRNA function by CMV $2 \mathrm{~b}$ in conjunction with HcPro was overcome by Y-Sat.

\section{Y-Sat siRNAs Bind to P19 and Sequester P19 from $h$ pGUS-Derived siRNAs}

As shown in Figure 1, Y-Sat-derived siRNAs were highly abundant in the infected plants. This led us to test if the satRNAderived siRNAs (sat-siRNAs) might bind and sequester the VSRs. RNA immunoprecipiation (RNA-IP) was performed on the $N$. benthamiana tissues agro-infiltrated with GUS $+h p G U S+P 19$ using the P19 antibody ( $\alpha$-P19). Northern analysis of the P19-bound sRNAs showed that Y-Sat-siRNAs were present at high levels in the $\alpha$-P19 RNA-IP from the Q-CMV+Y-Satinfected tissues (Figure 5A, middle-right panel). In contrast, the amount of $h p G U S$-derived siRNAs in the $\alpha$-P19 RNAIP was dramatically reduced compared to that in the plants 
A

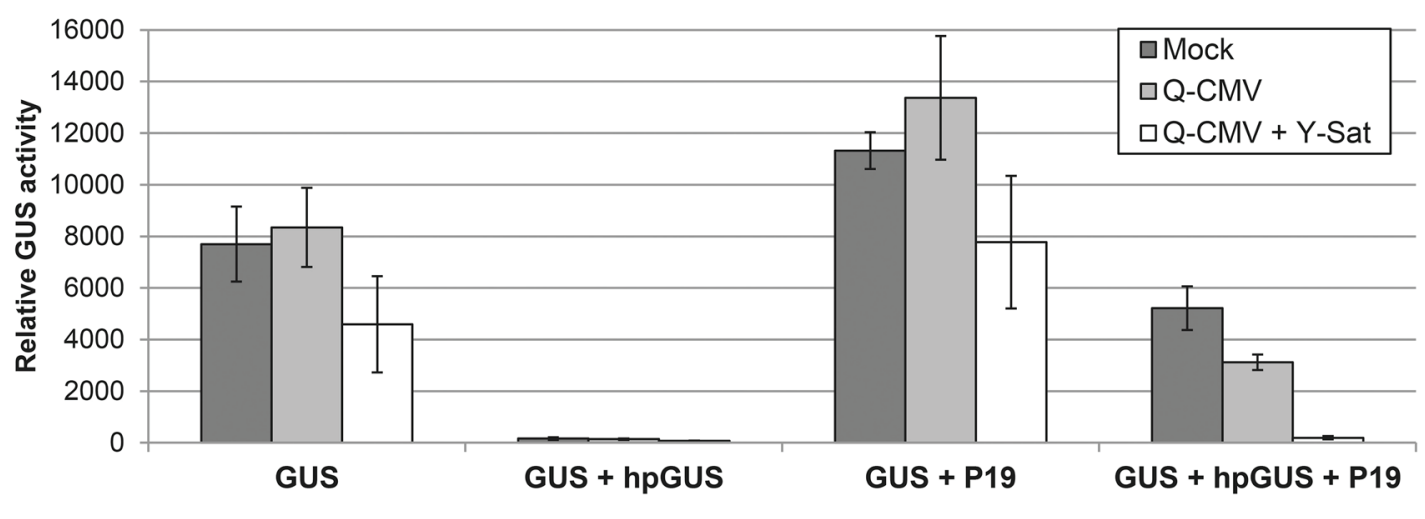

B
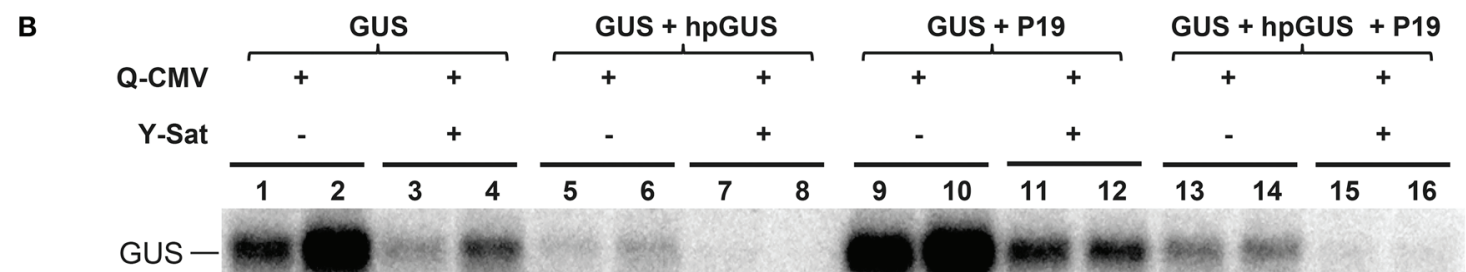

CMV RNA 3a-

Y-Sat -
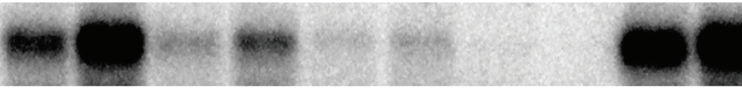

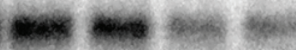

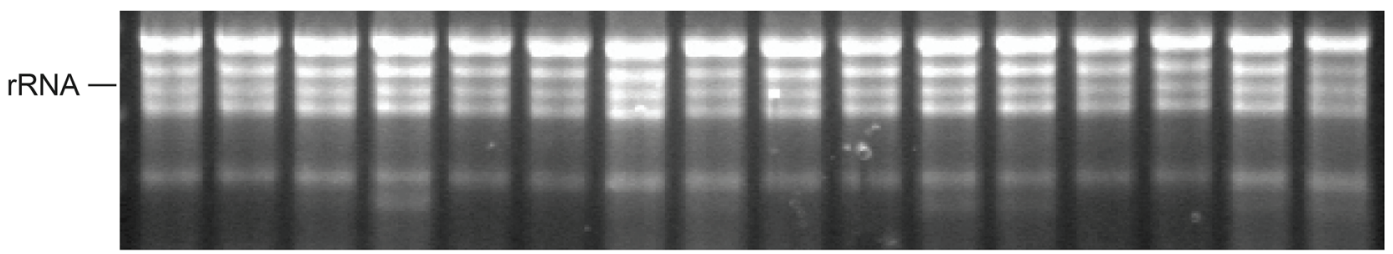

C

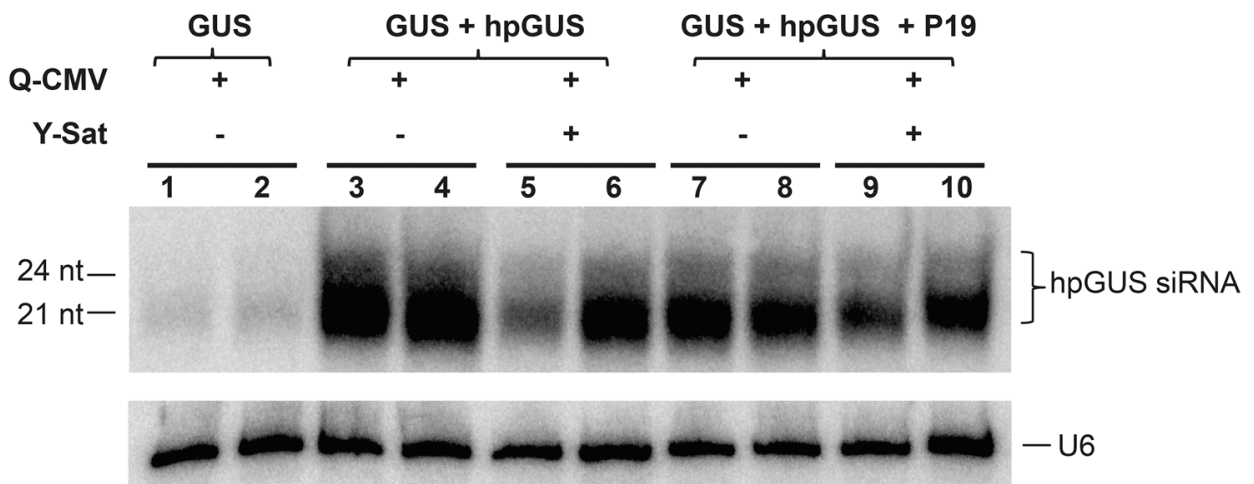

FIGURE 3 | The suppression of hpRNA-induced GUS silencing by agro-infiltrated P19 is released by CMV+Y-Sat infection. (A) MUG assay of GUS expression in Mock, Q-CMV and Q-CMV+Y-Sat-infected (17 days post incoculation) $N$. benthmaiana leaves infiltrated with the indicated constructs at $5 \mathrm{dpa}$. (B) Northern blot hybridization analysis of GUS expression in a subset of the samples described in (A). Five microgram of total RNA was hybridized with an antisense GUS probe to detect GUS mRNA levels (top panel), a CMV RNA 3a probe to measure CMV levels (second panel) and a Y-Sat probe to measure Y-Sat levels (third panel) in the agroinfiltrated tissues. Ethidium bromide-stained rRNA bands were used as loading control (bottom panel). (C) Northern blot hybridization analysis of small RNAs in a subset of the samples described in (A). Ten microgram of total RNA each was hybridized with a RNA probe corresponding to the dsRNA stem region of hpGUS to detect hpGUS-derived siRNA (top panel) and a U6 probe to show loading control (bottom panel). The sizes of the detected siRNAs are indicated. As can be seen, the level of hpGUS-derived siRNAs is not significantly affected by Q-CMV Y-Sat infection. 
A
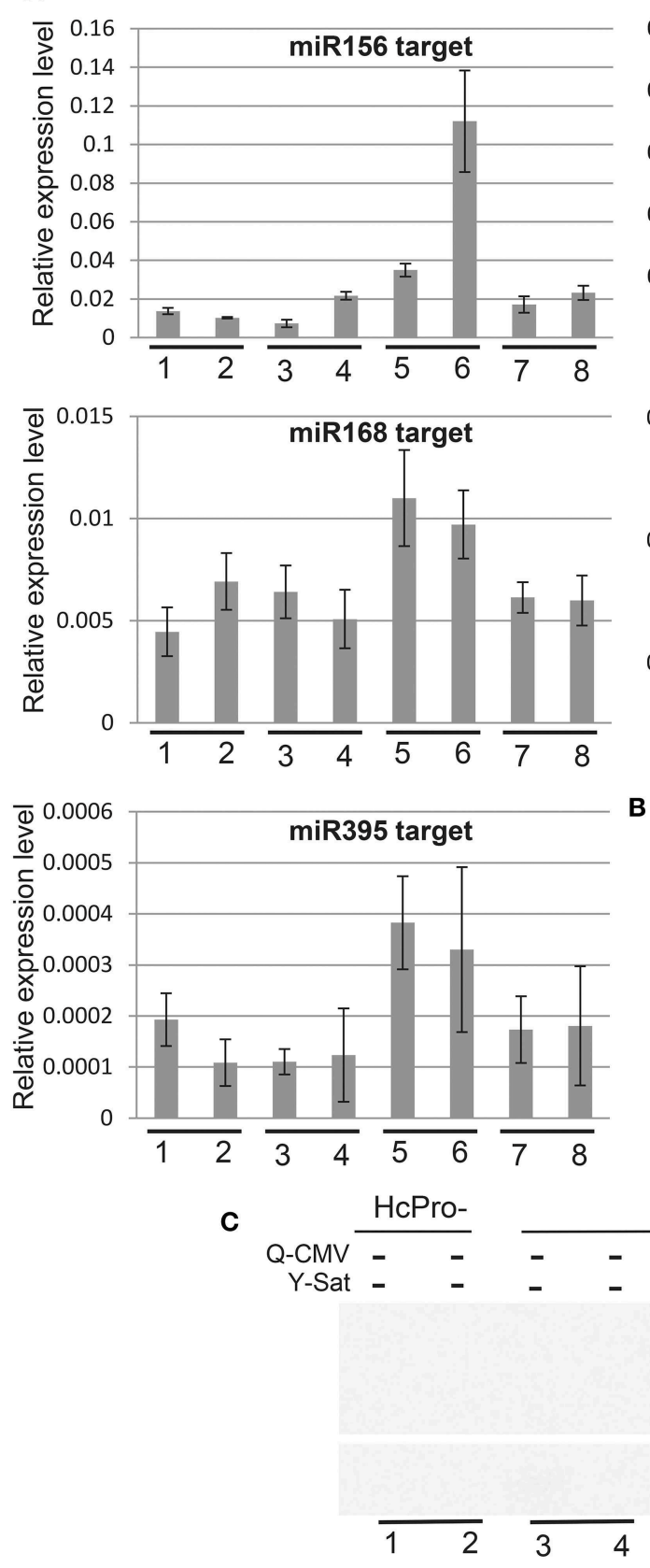

FIGURE 4 | The increased expression of miRNA target genes by CMV infection in HcPro-expressing $\boldsymbol{N}$. tabacum plants is reversed in the presence Y-Sat. (A) qRT-PCR analysis of predicted target genes for miR156, miR160, miR168, miR172, and miR395. The EF1 $\alpha$ gene was used as the internal reference. (B) qRT-PCR analysis of
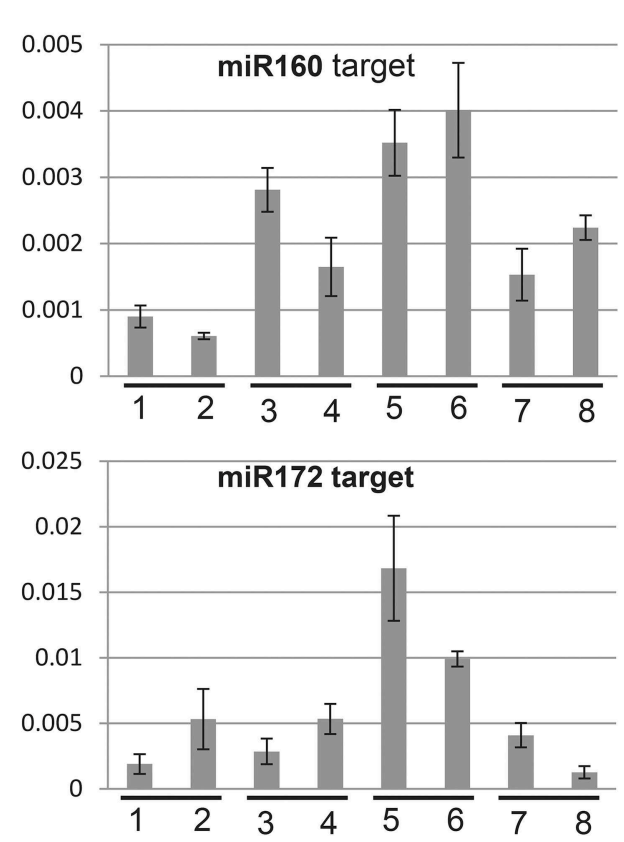

B 0.35

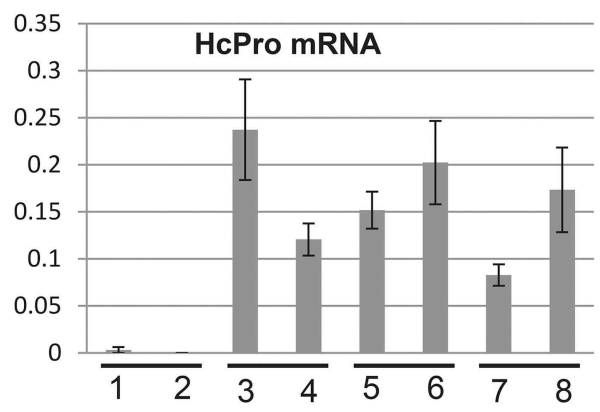

HcPro+
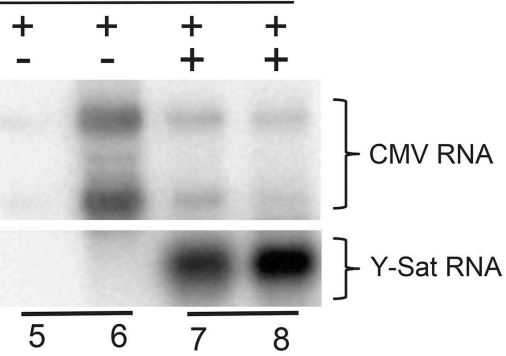

HcPro expression in the HcPro plants. (C) Northern blot analysis of Q-CMV and Y-Sat levels in the transgenic HcPro N. tabacum plants used for qRT-PCR analysis. HcPro+, N. tabacum plants containing the HcPro transgene; HcPro-, N. tabacum segregants containing no HcPro transgene.

infected by Q-CMV alone without Y-Sat (Figure 5A, top-right panel and Figure 5B). These results indicate that P19 suppresses the silencing of GUS expression via binding to the hpGUS-derived siRNAs, and that in the presence of Y-Sat, the abundant Y-Sat-derived siRNAs bind to P19 reduce its capacity to bind hpGUS-derived siRNAs. As a result, Y-Sat infection allowed more $h p G U S$-derived siRNAs to be available for directing GUS silencing. We did not pursue an RNA-IP assay for the binding of Y-Sat siRNAs to VSR $2 b$ due to the unavailability of $2 \mathrm{~b}$ antibody. However, from the previous studies showing that $2 \mathrm{~b}$ also suppresses RNA silencing by binding sRNAs (Vargason et al., 2003; Baulcombe and Molnar, 


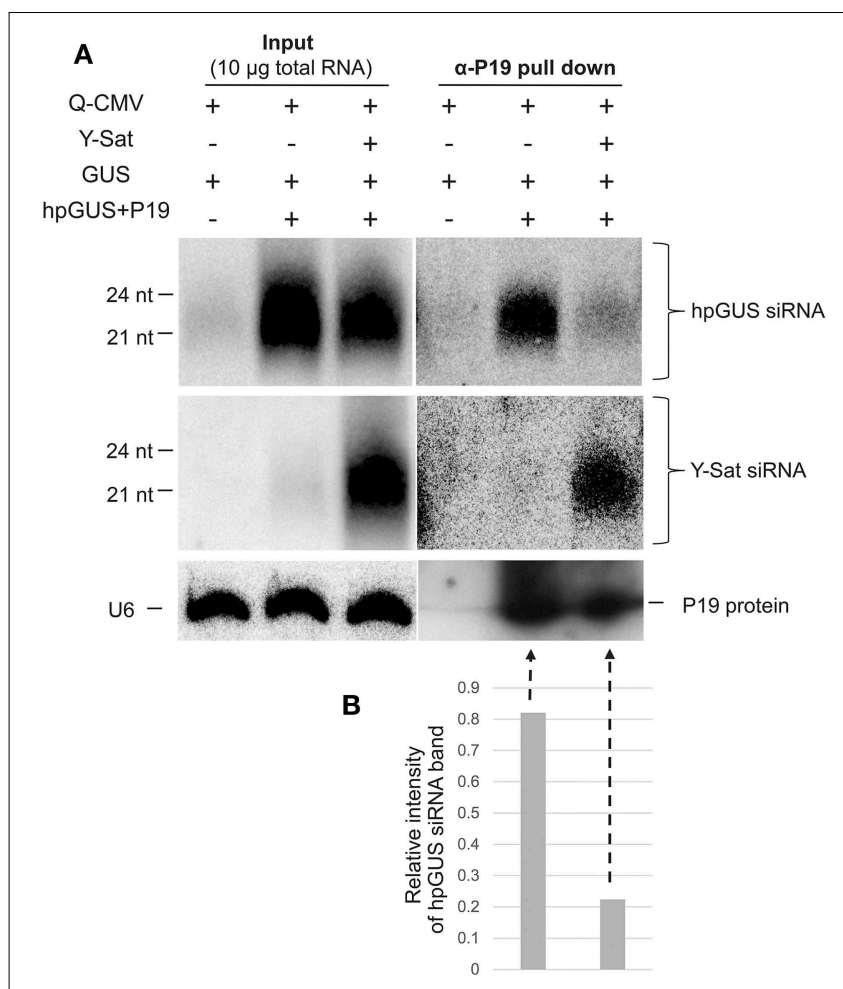

FIGURE 5 | RNA immunoprecipitation assay shows reduced binding of hpGUS-derived siRNAs to P19 in the presence of Y-Sat. (A) The amounts of GUS and Y-Sat siRNAs from the input (left three lanes) and RNA-IP (right three lanes) were determined by northern blot hybridization, which showed a strong reduction of hpGUS-derived siRNAs (top right panel) and abundant Y-Sat-derived siRNAs (middle right panel) in the presence of Y-Sat. The amounts of P19 protein in the RNA-IP pull-down product are indicated by Western blot (bottom right panel). The sizes of hpGUS- and Y-Sat-derived siRNAs are indicated. U6 small nuclear RNA hybridized by the U6 probe described above was used as a loading control for the input samples (bottom left panel). (B) Relative intensity of the $\alpha$-P19 pull-down hpGUS siRNA band (the last two lanes of the top panel) after normalization against the P19 protein band (the last two lanes of the bottom panel).

2004; Gonzalez et al., 2012), it is expected that the abundant Y-Sat-derived siRNAs would bind to $2 \mathrm{~b}$ and reduces the amount of $2 \mathrm{~b}$-bound $h p G U S$-derived siRNAs, enhancing GUS silencing in the $N$. benthamiana tissues agro-infiltrated with GUS+hpGUS+2b.

\section{Discussion}

A previous study on defective interfering RNAs (DI-RNAs) associated with Tomato bushy stunt tombusvirus (TBSV) has shown that abundant siRNAs derived from the DI-RNAs can saturate TBSV-encoded P19 VSR and elevate the levels of free TBSVderived siRNAs (Havelda et al., 2005). These free TBSV-derived siRNAs in the presence of the DI-RNAs have been proposed to play a role in directing silencing against the helper virus, thereby reducing TBSV accumulation and symptom severity (Havelda et al., 2005). A recent study on a CMV satRNA has also implicated increased anti-helper virus RNA silencing and reduced helper virus accumulation in satRNA-mediated symptom attenuation (Hou et al., 2011). This study shows that satRNA reduces the accumulation of VSR $2 \mathrm{~b}$. Because $2 \mathrm{~b}$ interferes with the function of viral sRNAs, it is likely that satRNA enhances anti-viral silencing (Hou et al., 2011) which in turn reduces the accumulation of CMV helper virus.

Whereas increased antiviral silencing and reduced helper virus accumulation as proposed in the two studies may well play a role in satRNA-mediated symptom attenuation, our results suggest an alternative mechanism by which satRNAs reduce helper virus-caused symptoms. Similar to the DI RNA scenario, satRNA-derived siRNAs bind to and saturate VSRs due to their high abundance in virus-infected tissues. However, in addition to promoting RNA silencing against the helper virus to reduce its accumulation, this minimizes the capacity of VSRs to bind host endogenous siRNAs and miRNAs. As a result, these endogenous siRNAs and miRNAs are free to form RISC with AGO proteins allowing normal sRNA-mediated gene regulation to occur in the host plants. Thus, developmental abnormalities, or symptoms, caused by the helper virus VSRs are minimized in the presence of satRNAs.

We have demonstrated that Y-Sat effectively reduced the ability of $2 \mathrm{~b}$ and P19 in suppressing the hpRNA-induced GUS silencing in $N$. benthamiana plants using the agro-infiltration-based transient expression assay. We have also shown that CMV infection increased the expression levels of miRNAs' target genes but in the presence of Y-Sat the expression levels of these target genes reverted to uninfected levels. Taken together, our data indicate that Y-Sat can impact on both the siRNA- and miRNA-directed RNA silencing pathways in the host plants. CMV $2 b$ was previously shown to bind both siRNAs and AGO proteins (Zhang et al., 2006; Duan et al., 2012; Hamera et al., 2012). However, in vivo suppression of RNA silencing requires its siRNA binding activity but is independent of its ability to interact directly with AGOs (Duan et al., 2012). We thus expect that binding and saturation of the 2b VSR by sat-siRNAs minimizes the ability of $2 b$ to bind host sRNAs and suppress host sRNA-directed RNA silencing, which would restore host sRNA-mediated gene regulation that would benefit normal plant development resulting in attenuated viral symptoms.

It is interesting to note that CMV infection alone seemed to affect the function of the ago-infiltrated $2 \mathrm{~b}$ and P19VSRs. There is a reduction in GUS expression in the CMV-infected in comparison to uninfected plants in GUS+hpGUS+2binfiltrated (Figure 2A) as well as GUS $+h p G U S+P 19$-infiltrated (Figure 3A) tissues. A possible explanation is that CMV-derived siRNAs, although less abundant than Y-Sat-derived siRNAs, also bind to the VSRs reducing their capacity to suppress hpRNAdirected GUS silencing. This result could imply that viral siRNAs not only play a role in directing silencing against the viruses, but also contribute to the minimization of VSR's interference with host plant sRNA functions.

satRNA-derived siRNAs have the potential to compete with host plant-encoded sRNAs for endogenous RNA silencing machineries such as DCLs and AGO proteins, which in turn could affect host sRNA-directed gene regulation. Thus, the high 
abundance of satRNA siRNAs could both reduce symptoms by binding to VSRs and exacerbate symptoms by competing for host silencing machineries. However, we have shown here and previously (Shen et al., 2014) that the high abundance of Y-Sat siRNAs does not seem to affect the biogenesis of host sRNAs in Nicotiana plants. Furthermore, in the absence of a strong VSR and visible helper virus-caused symptoms (namely in the case of the infection of $N$. benthamiana with Q-CMV which encodes a weak $2 b$ ), host sRNA-directed silencing and plant growth are not affected by the high level of Y-Sat siRNAs (Shen et al., 2014). Why host silencing machineries are not saturated by the abundant satRNA siRNAs remains to be further understood, but these results suggest that satRNA-derived siRNAs affect host sRNA functions mainly through interacting with VSRs.

In conclusion, our results suggest that satRNAs are likely to attenuate the symptoms via their abundant siRNAs, which bind helper virus-encoded VSRs minimizing their interference with the endogenous siRNA and miRNA pathways as well as the antiviral silencing pathways as proposed in the previous studies. This model could apply to other viral or subviral RNAs that give rise to high levels of siRNAs in infected host cells, for example, DI RNAs. DI RNAs are a truncated form of their associated helper viral genomes and, like satRNAs, also depend on the helper virus for replication and can also attenuate helper virus-induced symptoms (Simon et al., 2004). Unlike satRNAs that occur mostly in plants, DI RNAs are widespread and associated with both plant viruses and animal viruses. Because animal viruses also encode VSRs such as the B2 protein encoded by Flock House virus (FHV) (Aliyari et al., 2008), it would be valuable to examine if DI RNAs modulate animal viral symptoms through a similar mechanism.

\section{References}

Aliyari, R., Wu, Q. F., Li, H. W., Wang, X. H., Li, F., Green, L. D., et al. (2008). Mechanism of induction and suppression of antiviral immunity directed by virus-derived small RNAs in Drosophila. Cell Host Microbe 4, 387-397. doi: 10.1016/j.chom.2008.09.001

Baulcombe, D. C., and Molnar, A. (2004). Crystal structure of p19-a universal suppressor of RNA silencing. Trends Biochem. Sci. 29, 279-281. doi: 10.1016/j.tibs.2004.04.007

Baulcombe, D. C., Saunders, G. R., Bevan, M. W., Mayo, M. A., and Harrison, B. D. (1986). Expression of biologically-active viral satellite RNA from the nuclear genome of transformed plants. Nature 321, 446-449. doi: 10.1038/321446a0

Brigneti, G., Voinnet, O., Li, W. X., Ji, L. H., Ding, S. W., and Baulcombe, D. C. (1998). Viral pathogenicity determinants are suppressors of transgene silencing in Nicotiana benthamiana. EMBO J. 17, 6739-6746. doi: 10.1093/emboj/17.22.6739

Chapman, E. J., Prokhnevsky, A. I., Gopinath, K., Dolja, V. V., and Carrington, J. C. (2004). Viral RNA silencing suppressors inhibit the microRNA pathway at an intermediate step. Gene Dev. 18, 1179-1186. doi: 10.1101/gad.1201204

Chen, S., Helliwell, C. A., Wu, L. M., Dennis, E. S., Upadhyaya, N. M., Zhang, R., et al. (2005). A novel T-DNA vector design for selection of transgenic lines with simple transgene integration and stable transgene expression. Funct. Plant Biol. 32, 671-681. doi: 10.1071/FP05072

Ding, S. W., Shi, B. J., Li, W. X., and Symons, R. H. (1996). An interspecies hybrid RNA virus is significantly more virulent than either parental virus. Proc. Natl. Acad. Sci. U.S.A. 93, 7470-7474. doi: 10.1073/pnas.93.15.7470

Du, Z. Y., Chen, A. Z., Chen, W. H., Liao, Q. S., Zhang, H. M., Bao, Y. M., et al. (2014). Nuclear-cytoplasmic partitioning of Cucumber mosaic virus protein $2 \mathrm{~b}$ determines the balance between its roles as a virulence determinant and an RNA-silencing suppressor. J. Virol. 88, 5228-5241. doi: 10.1128/JVI.00284-14
If so, potential DI RNA-related strategies could be developed against animal viral diseases in the future.

\section{Funding}

WS was funded by a China Scholarship Council scholarship, grants from MOA's Public Benefit Research Foundation of China (201203076) and Chongqing Postdoctoral Science Foundation (Rc201423) and the Program for Changjiang Scholars and Innovative Research Team in University (IRT0976), and MW was supported by an Australian Research Council Future Fellowship (FT0991956).

\section{Acknowledgments}

We would like to thank Craig Wood and Christopher Cazzonelli for helpful discussion and technical advice on agroinfiltration assays, Herman Bertus Scholthof for P19 antibody, Chikara Masuta for the Y-Sat infectious clone, Kenzo Nakamura for the GUS construct pIG121Hm, Vickie Vance, and Peter Waterhouse for the HcPro transgenic plants, Carl Davies for photography, and Zhiyou Du for critical reading of the manuscript. We also thank Joanne Lee for mapping the CMV and Y-Sat sRNAs.

\section{Supplementary Material}

The Supplementary Material for this article can be found online at: http://journal.frontiersin.org/article/10.3389/fpls.2015. 00281/abstract

Duan, C. G., Fang, Y. Y., Zhou, B. J., Zhao, J. H., Hou, W. N., Zhu, H., et al. (2012). Suppression of Arabidopsis ARGONAUTE1-mediated slicing, transgene-induced RNA silencing, and DNA methylation by distinct domains of the Cucumber mosaic virus 2b protein. Plant Cell 24, 259-274. doi: $10.1105 /$ tpc.111.092718

Ebhardt, H. A., and Unrau, P. J. (2009). Characterizing multiple exogenous and endogenous small RNA populations in parallel with subfemtomolar sensitivity using a streptavidin gel-shift assay. RNA 15, 724-731. doi: 10.1261/rna.1235109

Garcia-Arenal, F., and Palukaitis, P. (1999). Structure and functional relationships of satellite RNAs of Cucumber mosaic virus. Curr. Top. Microbiol. 239, 37-63. doi: 10.1007/978-3-662-09796-0_3

Gerlach, W. L., Llewellyn, D., and Haseloff, J. (1987). Construction of a plant disease resistance gene from the satellite RNA of Tobacco ringspot virus. Nature 328, 802-805. doi: 10.1038/328802a0

Gonzalez, I., Rakitina, D., Semashko, M., Taliansky, M., Praveen, S., Palukaitis, P., et al. (2012). RNA binding is more critical to the suppression of silencing function of Cucumber mosaic virus $2 \mathrm{~b}$ protein than nuclear localization. RNA 18, 771-782. doi: 10.1261/rna.031260.111

Hamera, S., Song, X. G., Su, L., Chen, X. Y., and Fang, R. X. (2012). Cucumber mosaic virus suppressor $2 \mathrm{~b}$ binds to AGO4-related small RNAs and impairs AGO4 activities. Plant J. 69, 104-115. doi: 10.1111/j.1365-313X.2011. 04774.x

Harrison, B. D., Mayo, M. A., and Baulcombe, D. C. (1987). Virus-resistance in transgenic plants that express Cucumber mosaic virus satellite RNA. Nature 328, 799-802. doi: 10.1038/328799a0

Havelda, Z., Hornyik, C., Valoczi, A., and Burgyan, J. (2005). Defective interfering RNA hinders the activity of a Tombusvirus-encoded posttranscriptional gene silencing suppressor. J. Virol. 79, 450-457. doi: 10.1128/JVI.79.1.450-457.2005

Hou, W. N., Duan, C. G., Fang, R. X., Zhou, X. Y., and Guo, H. S. (2011). Satellite $\mathrm{RNA}$ reduces expression of the $2 \mathrm{~b}$ suppressor protein resulting in the 
attenuation of symptoms caused by Cucumber mosaic virus infection. Mol. Plant Pathol. 12, 595-605. doi: 10.1111/j.1364-3703.2010.00696.x

$\mathrm{Hu}$, C. C., Hsu, Y. H., and Lin, N. S. (2009). Satellite RNAs and satellite viruses of plants. Viruses 1, 1325-1350. doi: 10.3390/v1031325

Ishikawa, A. (2009). The Arabidopsis G-Protein beta-subunit is required for defense response against Agrobacterium tumefaciens. Biosci. Biotechnol. Biochem. 73, 47-52. doi: 10.1271/bbb.80449

Jay, F., Wang, Y., Yu, A., Taconnat, L., Pelletier, S., Colot, V., et al. (2011). Misregulation of AUXIN RESPONSE FACTOR 8 underlies the developmental abnormalities caused by three distinct viral silencing suppressors in Arabidopsis. PLoS Pathog 7: e1002035. doi: 10.1371/journal.ppat.1002035

Kasschau, K. D., Xie, Z., Allen, E., Llave, C., Chapman, E. J., Krizan, K. A., et al. (2003). P1/HC-Pro, a viral suppressor of RNA silencing, interferes with Arabidopsis development and miRNA function. Dev. Cell 4, 205-217. doi: 10.1016/S1534-5807(03)00025-X

Lakatos, L., Csorba, T., Pantaleo, V., Chapman, E. J., Carrington, J. C., Liu, Y. P., et al. (2006). Small RNA binding is a common strategy to suppress RNA silencing by several viral suppressors. EMBO J. 25, 2768-2780. doi: 10.1038/sj.emboj.7601164

Lewsey, M., Robertson, F. C., Canto, T., Palukaitis, P., and Carr, J. P. (2007). Selective targeting of miRNA-regulated plant development by a viral countersilencing protein. Plant J. 50, 240-252. doi: 10.1111/j.1365-313X.2007.03042.x

Liu, L., and Lomonossoff, G. P. (2002). Agroinfection as a rapid method for propagating Cowpea mosaic virus-based constructs. J. Virol. Methods 105, 343-348. doi: 10.1016/S0166-0934(02)00121-0

Masuta, C., and Takanami, Y. (1989). Determination of sequence and structural requirements for pathogenicity of a Cucumber mosaic virus satellite RNA (Y-satRNA). Plant Cell 1, 1165-1173. doi: 10.1105/tpc.1.12.1165

Moriones, E., Diaz, I., Rodriguezcerezo, E., Fraile, A., and Garciaarenal, F. (1992). Differential interactions among strains of Tomato aspermy virus and satellite RNAs of Cucumber mosaic virus. Virology 186, 475-480. doi: 10.1016/00426822(92)90012-E

Ohta, S., Mita, S., Hattori, T., and Nakamura, K. (1990). Construction and expression in Tobacco of a $\beta$-Glucuronidase (GUS) reporter gene dontaining an intron within the coding sequence. Plant Cell Physiol. 31, 805-813.

Omarov, R., Sparks, K., Smith, L., Zindovic, J., and Scholthof, H. B. (2006). Biological relevance of a stable biochemical interaction between the Tombusvirusencoded P19 and short interfering RNAs. J. Virol. 80, 3000-3008. doi: 10.1128/JVI.80.6.3000-3008.2006

Palukaitis, P., Roossinck, M. J., Dietzgen, R. G., and Francki, R. I. B. (1992). Cucumber mosaic virus. Adv. Virus Res. 41, 281-348. doi: 10.1016/S00653527(08)60039-1

Piazzolla, P., Tousignant, M. E., and Kaper, J. M. (1982). Cucumber mosaic virusassociated RNA 5 IX. The overtaking of viral RNA synthesis by CARNA 5 and dsCARNA 5 in tobacco. Virology 122, 147-157. doi: 10.1016/00426822(82)90384-1

Pumplin, N., and Voinnet, O. (2013). RNA silencing suppression by plant pathogens: defence, counter-defence and counter-counter-defence. Nat. Rev. Microbiol. 11, 745-760. doi: 10.1038/nrmicro3120

Roossinck, M. J., Sleat, D., and Palukaitis, P. (1992). Satellite RNAs of plant viruses: structures and biological effects. Microbiol. Rev. 56, 265-279.

Ruiz-Ferrer, V., and Voinnet, O. (2009). Roles of plant small RNAs in biotic stress responses. Annu. Rev. Plant Biol. 60, 485-510. doi: 10.1146/annurev.arplant.043008.092111

Sambrook, J., Fritsch, E., and Maniatis, T. (1989). Molecular-Cloning - A Laboratory Manual, 2nd Edn. Cold Spring Harbor, NY: Cold Spring Harbor Laboratory Press.

Shen, W. X., Smith, N. A., Zhou, C. Y., and Wang, M. B. (2014). Highlevel accumulation of exogenous small RNAs not affecting endogenous small RNA biogenesis and function in plants. J. Integr. Agric. 13, 1017-1023. doi: 10.1016/S2095-3119(13)60525-0

Shi, B. J., Palukaitis, P., and Symons, R. H. (2002). Differential virulence by strains of Cucumber mosaic virus is mediated by the $2 \mathrm{~b}$ gene. Mol. Plant Microbe Interact. 15, 947-955. doi: 10.1094/MPMI.2002.15.9.947

Shimura, H., and Pantaleo, V. (2011). Viral induction and suppression of RNA silencing in plants. Biochim. Biophys. Acta 1809, 601-612. doi: 10.1016/j.bbagrm.2011.04.005

Shimura, H., Pantaleo, V., Ishihara, T., Myojo, N., Inaba, J., Sueda, K., et al. (2011). A viral satellite RNA induces yellow symptoms on tobacco by targeting a gene involved in chlorophyll biosynthesis using the RNA silencing machinery. PLoS Pathog. 7:e1002021. doi: 10.1371/journal.ppat.1002021

Simon, A. E., Roossinck, M. J., and Havelda, Z. (2004). Plant virus satellite and defective interfering RNAs: new paradigms for a new century. Annu. Rev. Phytopathol. 42, 415-437. doi: 10.1146/annurev.phyto.42.040803.140402

Smith, N. A., Eamens, A. L., and Wang, M. B. (2011). Viral small interfering RNAs target host genes to mediate disease symptoms in plants. PLoS Pathog. 7:e1002022. doi: 10.1371/journal.ppat.1002022

Soards, A. J., Murphy, A. M., Palukaitis, P., and Carr, J. P. (2002). Virulence and differential local and systemic spread of Cucumber mosaic virus in tobacco are affected by the CMV 2b protein. Mol. Plant Microbe Interact. 15, 647-653. doi: 10.1094/MPMI.2002.15.7.647

Takanami, Y. (1981). A striking change in symptoms on cucumber mosaic virusinfected tobacco plants induced by a satellite RNA. Virology 109, 120-126. doi: 10.1016/0042-6822(81)90476-1

Vargason, J. M., Szittya, G., Burgyan, J., and Hall, T. M. (2003). Size selective recognition of siRNA by an RNA silencing suppressor. Cell 115, 779-811. doi: 10.1016/S0092-8674(03)00984-X

Voinnet, O. (2009). Origin, biogenesis, and activity of plant microRNAs. Cell 136, 669-687. doi: 10.1016/j.cell.2009.01.046

Voinnet, O., Pinto, Y. M., and Baulcombe, D. C. (1999). Suppression of gene silencing: a general strategy used by diverse DNA and RNA viruses of plants. Proc. Natl. Acad. Sci. U.S.A. 96, 14147-14152. doi: 10.1073/pnas.96.24.14147

Wang, M. B., Bian, X. Y., Wu, L. M., Liu, L. X., Smith, N. A., Isenegger, D., et al. (2004). On the role of RNA silencing in the pathogenicity and evolution of viroids and viral satellites. Proc. Natl. Acad. Sci. U.S.A. 101, 3275-3280. doi: 10.1073/pnas.0400104101

Wang, M. B., Helliwell, C. A., Wu, L. M., Waterhouse, P. M., Peacock, W. J., and Dennis, E. S. (2008). Hairpin RNAs derived from RNA polymerase II and polymerase III promoter-directed transgenes are processed differently in plants. RNA 14, 903-913. doi: 10.1261/rna.760908

Wang, M. B., and Waterhouse, P. M. (2000). High-efficiency silencing of a betaglucuronidase gene in rice is correlated with repetitive transgene structure but is independent of DNA methylation. Plant Mol. Biol. 43, 67-82. doi: 10.1023/A:1006490331303

Wang, M. B., Wesley, S. V., Finnegan, E. J., Smith, N. A., and Waterhouse, P. M. (2001). Replicating satellite RNA induces sequence-specific DNA methylation and truncated transcripts in plants. RNA 7, 16-28. doi: $10.1017 /$ S1355838201001224

Wood, C. C., Petrie, J. R., Shrestha, P., Mansour, M. P., Nichols, P. D., Green, A. G., et al. (2009). A leaf-based assay using interchangeable design principles to rapidly assemble multistep recombinant pathways. Plant Biotechnol. J. 7, 914-924. doi: 10.1111/j.1467-7652.2009.00453.x

Wu, G., and Kaper, J. M. (1995). Competition of viral and satellite RNAs of Cucumber mosaic virus for replication in vitro by viral RNA-dependent RNA polymerase. Res. Virol. 146, 61-67. doi: 10.1016/0923-2516(96)80590-5

Ye, J., Qua, J., Zhang, J. F., Geng, Y. F., and Fang, R. X. (2009). A critical domain of the Cucumber mosaic virus $2 \mathrm{~b}$ protein for RNA silencing suppressor activity. FEBS Lett. 583, 101-106. doi: 10.1016/j.febslet.2008.11.031

Zamore, P. D. (2001). RNA interference: listening to the sound of silence. Nat. Struct. Biol. 8, 746-750. doi: 10.1038/nsb0901-746

Zhang, L., Hanada, K., and Palukaitis, P. (1994). Mapping local and systemic symptom determinants of Cucumber mosaic cucumovirus in tobacco. J. Gen. Virol. 75, 3185-3191. doi: 10.1099/0022-1317-75-11-3185

Zhang, X. R., Yuan, Y. R., Pei, Y., Lin, S. S., Tuschl, T., Patel, D. J., et al. (2006). Cucumber mosaic virus-encoded $2 \mathrm{~b}$ suppressor inhibits Arabidopsis Argonaute1 cleavage activity to counter plant defense. Gene Dev. 20, 3255-3268. doi: 10.1101/gad.1495506

Conflict of Interest Statement: The authors declare that the research was conducted in the absence of any commercial or financial relationships that could be construed as a potential conflict of interest.

Copyright (๑ 2015 Shen, Au, Shi, Smith, Dennis, Guo, Zhou and Wang. This is an open-access article distributed under the terms of the Creative Commons Attribution License (CC BY). The use, distribution or reproduction in other forums is permitted, provided the original author(s) or licensor are credited and that the original publication in this journal is cited, in accordance with accepted academic practice. No use, distribution or reproduction is permitted which does not comply with these terms. 